Itinéraires Itinéraires

Littérature, textes, cultures

\title{
Jean Paulhan et l'obsession du plan
}

Jean Paulhan and the Obsession with the Plan

\section{Camille Koskas}

\section{(2) OpenEdition}

Journals

Édition électronique

URL : http://journals.openedition.org/itineraires/7467

DOI : $10.4000 /$ itineraires. 7467

ISSN : 2427-920X

Éditeur

Pléiade

\section{Référence électronique}

Camille Koskas, « Jean Paulhan et l'obsession du plan », Itinéraires [En ligne], 2020-1 | 2020, mis en ligne le 02 octobre 2020, consulté le 13 novembre 2020. URL : http://journals.openedition.org/ itineraires/7467 ; DOI : https://doi.org/10.4000/itineraires.7467

Ce document a été généré automatiquement le 13 novembre 2020.

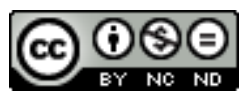

Itinéraires est mis à disposition selon les termes de la licence Creative Commons Attribution - Pas d'Utilisation Commerciale - Pas de Modification 4.0 International. 


\title{
Jean Paulhan et l'obsession du plan
}

\author{
Jean Paulhan and the Obsession with the Plan
}

\author{
Camille Koskas
}

Nous tenons à remercier l'équipe de l'IMEC et Claire Paulhan, qui nous ont donné accès au fonds Paulhan et aux documents sur lesquels cet article s'appuie, et qui nous ont autorisée à reproduire ici ces images.

1 Les études, peu nombreuses, qui se sont consacrées aux récits paulhaniens ${ }^{1}$, ont insisté sur leur caractère hermétique, déstabilisant. Comme Michel Murat l'a souligné, ces difficultés tiennent en bonne part à leur ambiguïté générique : ni roman, ni nouvelle, ni pure fiction, ni autobiographie. De même, les frontières entre textes de nature essayistique et récits sont souvent poreuses. En raison de cette ambiguïté générique, le lecteur est confronté à la difficulté d'appréhender et d'identifier la nature des nombreuses sections et sous-sections qui se multiplient au sein des textes. Jean-Yves Tadié note qu'au moment du déploiement des dernières grandes fresques de la littérature européenne (Proust, Mann, Musil), Paulhan se détourne de la forme longue pour se concentrer sur des récits très courts, très fragmentés, découpés en unités qui dépassent rarement cinq pages (Tadié 1994 : 102). Peut-on parler de chapitres, malgré cette brièveté ? Faut-il préférer le terme de sections, voire de fragments, tant Paulhan s'ingénie parfois à escamoter les liens qui relient chacune de ces unités? Jean-Yves Tadié utilise le terme de " conte ${ }^{2}$ » pour qualifier les 21 unités qui composent Les Causes Célèbres, terme qui semble sous-entendre que celles-ci entretiennent une relative autonomie les unes par rapport aux autres, ou du moins, peuvent se prêter à différentes lectures, selon qu'elles s'appréhendent de manière indépendante (l'une d'entre elles s'intitule d'ailleurs "Une espèce d'île ») ou qu'elles soient inscrites, à travers le parcours du lecteur, dans le fil d'un récit. C'est toujours chez Paulhan la structure qui commande le sens, ou du moins qui ajoute et modifie le sens ${ }^{3}$.

2 La lecture des récits paulhaniens fait ainsi appel à la sagacité du lecteur qui doit reconstituer ces articulations manquantes, bien souvent effacées par l'auteur au cours de ces différents travaux sur les plans, pour approcher le sens global du texte. D'autre part, Paulhan mobilise différents types de dispositifs pour souligner et accentuer ces découpages, jouant sur les titres, les numérotations, les blancs, les changements de 
typographie. D'autres procédés peuvent être utilisés, plus rarement: comme l'a souligné Bernard Baillaud, qui propose dans sa thèse une analyse des titres utilisés par Paulhan dans Le Guerrier appliqué (Baillaud 2000 : 292-304), Paulhan se plaît à cultiver un effet d'attente chez son lecteur, qu'il fait durer en choisissant d'associer le titre à un élément situé plutôt à la fin d'une section qu'au début. Bernard Baillaud rappelle également que les premières éditions de ce texte (chez Sansot, en 1917) comptaient plusieurs exemplaires illustrés, à l'ouverture de chaque section, par des vignettes dessinées par Albert Uriet : leur fonction serait de constituer des «titres visuels » aux côtés des titres verbaux (Baillaud $2000: 298$ ). Nous sommes donc face à un auteur pour qui la segmentation, la fragmentation occupent une place essentielle aussi bien au niveau de la genèse de l'œuvre que du point de vue du rendu final du texte. Bernard Baillaud a bien souligné la difficulté à disposer le texte dans la genèse des Fleurs de Tarbes: l'agencement de ces différentes unités, leur ordonnancement, sont l'objet de remaniements multiples au sein de la phase d'écriture dont les documents d'archives rendent compte (Baillaud 2000 : 304). Nous entendons montrer ici que cette logique de construction à la fois ludique et rigoureuse constitue une clé possible de compréhension des récits paulhaniens, en examinant les différents types de dispositifs qui y sont mis en œuvre. Quel effet provoque cette disposition hypersegmentée sur le lecteur? Nous entendons également questionner cette inclination pour la forme courte au sein du projet d'écriture de Paulhan, qu'il s'agisse des récits mais aussi des textes de nature essayistique : comment le caractère extrêmement segmenté de ces textes entre$\mathrm{t}$-il en tension avec le désir très fort d'unité qui habite l'ensemble de l'œuvre?

\section{Des récits dominés par deux types d'organisation}

\section{La succession linéaire de différentes unités placées sur le même plan hiérarchique}

3 C'est le cas de Progrès en amour assez lents, texte écrit en 1916 dont le récit s'organise en douze sections, chiffrées et datées. C'est le cas également de La Métromanie ou les Dessous de la capitale (cinq sections titrées comptant deux pages maximum) ou du Guide d'un petit voyage en Suisse (1947), qui voit se succéder sept sections numérotées. Paulhan y relate, sur un mode ironique, le séjour fait en Suisse après la Libération. Le manuscrit reproduit dans l'édition de Bernard Baillaud (Paulhan [1947] 2006: 514) montre que chaque section s'ouvre sur un sommaire résumant les principaux mouvements $\mathrm{du}$ chapitre, éléments que Paulhan reprend dans la version définitive, en les redistribuant verticalement, comme une sorte de petit poème dans la tradition de l'épigraphe. La forme permet à Paulhan de jouer avec les ressources de la versification. La quatrième section s'ouvre ainsi sur le petit texte suivant :

Fréquentation des palaces

Les doubles portes et le verrou électrique

De Lausanne à Berne en passant par Zurich

L'amoureux de la boulangère (Paulhan [1947] 2006 : 336)

Le simple sommaire devient objet poétique, comme le montre la disposition verticale, mais aussi le choix de l'hendécasyllabe pour les deux vers centraux et la rime entre « électrique » et «Zurich». L'accumulation des noms de ville crée une impression de rapidité, semblant indiquer au lecteur que contrairement à un récit de voyage classique, ce n'est pas dans le trajet entre ces différentes villes que se situe l'intérêt du 
texte. La chute de ce quatrain crée une sorte de court-circuit (des palaces à la boulangerie), d'autant que le lecteur découvrira ensuite que l'amoureux de la boulangère annonçait en réalité une anecdote scatologique. Des liens se tissent aussi entre les sept épigraphes des différentes sections, accompagnant les mouvements de la narration. L'épigraphe de la première section s'achève ainsi sur ces mots : "Comment reconnaître les paysages inoubliables ", auxquels répond l'épigraphe de la dernière section: "Je découvre enfin des paysages inoubliables", jeu d'écho qui semble organiser le récit comme une quête (Paulhan [1947] 2006: 325 et 347). Enfin ces formules d'ouverture créent aussi des effets d'attente par rapport au texte des différentes sections qu'elles chapeautent, en redoublant le sens ou en le courtcircuitant de manière ironique. Comme on le voit, ce petit objet rayonne donc à différents niveaux du texte. Ce sont même vers d'autres textes de l'œuvre de Paulhan, hors des frontières de ce récit, que ces lignes font signe. "Progrès rapides de la vanité » (ces mots constituent le premier vers de l'épigraphe de la troisième section - Paulhan 2006 : 333) peut se lire comme un clin d'œil aux Progrès en amour assez lents (la vanité progresse plus vite que l'amour...) écrits trente ans plus tôt.

Un autre cas dans lequel Paulhan use de ce type de structure linéaire nous est fourni par Les Causes célèbres (1946), que nous évoquions plus haut et pour lequel nous disposons d'un copieux dossier d'archives. Nous l'avons dit, le statut de ces 21 textes est complexe : contes, fables ou apologues pour les critiques, écrits tantôt à la première personne, tantôt à la troisième personne, ils peuvent se lire de manière parfaitement indépendante les uns par rapport aux autres. Le seul indice de la cohérence du texte est proposé dans l'épigraphe, placée en tête du titre, et empruntée à un Manuel de Physique à l'usage des gens du monde : « Nous autres physiciens avons coutume de nommer "causes célèbres" celles des causes que nous décelons dont l'effet est paradoxal " (Paulhan [1946] 2006 : 275). C'est en effet ce jeu des renversements paradoxaux qui rapproche les différentes unités du récit, depuis l'homme du premier conte venu contempler un coucher de soleil, décrit avec force images très visuelles (débauche de couleurs et alliance des contraires qui font du ciel un fleuve enflammé), et dont le spectateur se révèle finalement aveugle, au maçon sanguinolent apparemment gravement blessé après un éboulis, dont on découvre que le sang qui le recouvre provient d'une main qu'il a découpée à une agonisante pour lui voler sa bague. Il y a donc une violence du corps, mais aussi un prosaïsme qui ressurgit volontiers à la clôture de chaque conte : le premier de ses récits se clôt aux cris de "la soupe est chaude" alors que l'aveugle suspend sa contemplation des cieux pour aller dîner; le troisième sur la mention des " chiottes ", le cinquième sur l'image horrifique de la main coupée. Tout se passe, peutêtre, comme si la violence du corps, la trivialité de ses fonctions, la présence de la sexualité, jaillissaient dans les interstices d'une structure bien huilée. Il y aurait évidemment une analyse fine à faire des liens entre le corps du texte et les titres des sections, les effets de surprise et d'attente qu'ils ménagent, mais nous préférons nous concentrer ici sur les éléments que mettent en lumière les dossiers d'archives déposés à l'Institut Mémoire de l'édition contemporaine (IMEC) ${ }^{4}$. Ceux-ci permettent de saisir les différentes étapes de cette architecture méticuleusement composée. Paulhan organise son texte grâce à différents types de dispositifs.

5 Premier type de dispositif, les grilles, organisées autour des titres de section, qui comprennent chacun une à deux cases. 
Figure 1. Plan des Causes célèbres : sections «Orpaillargues ", « Je sais ce que tu veux ", "La bonne soirée»

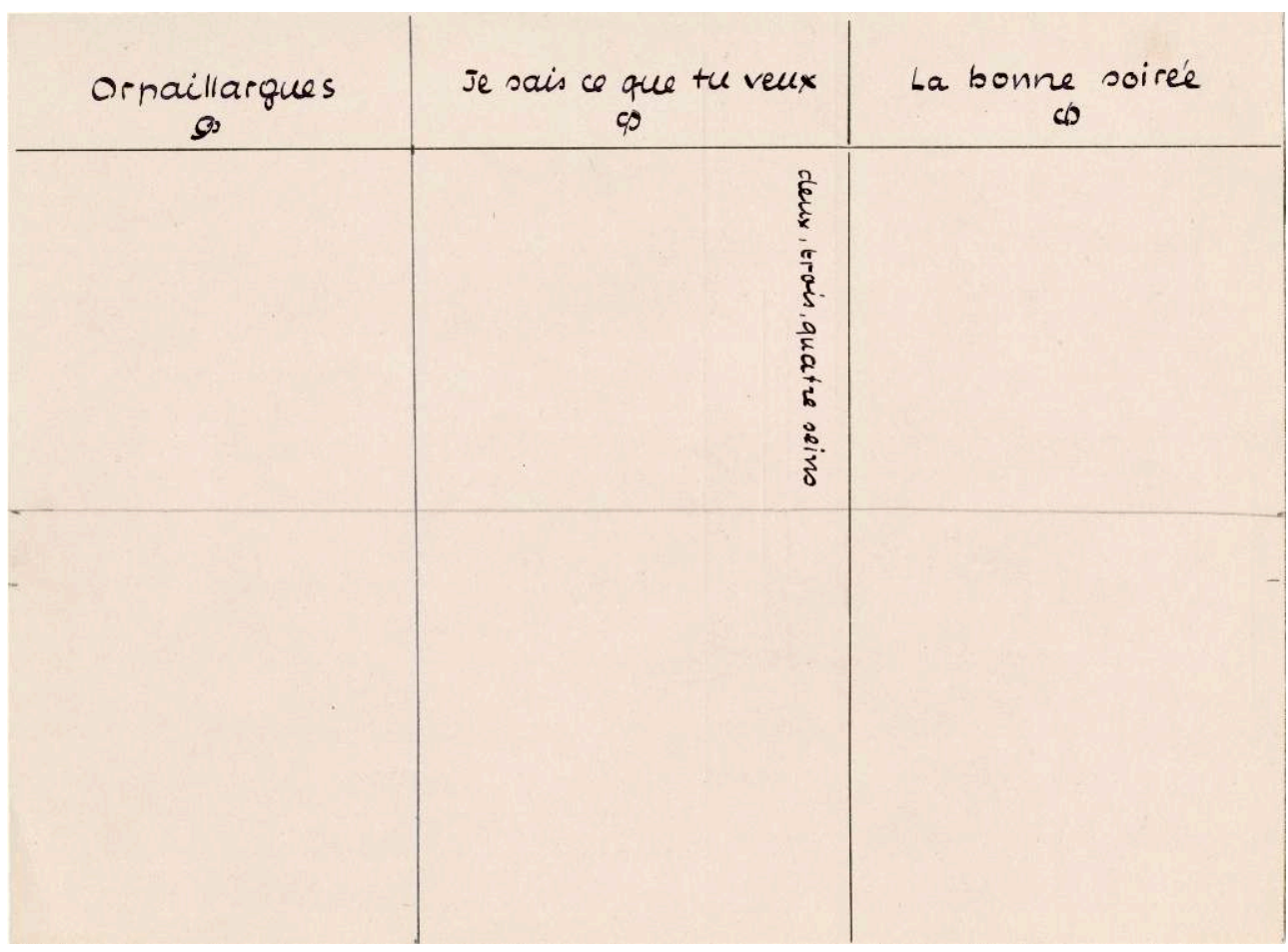

Source : fonds Paulhan, IMEC

Figure 2. Plan des Causes célèbres : sections « Les cigarettes ", " Jeanne est en retard ", " Pensée sans objet"

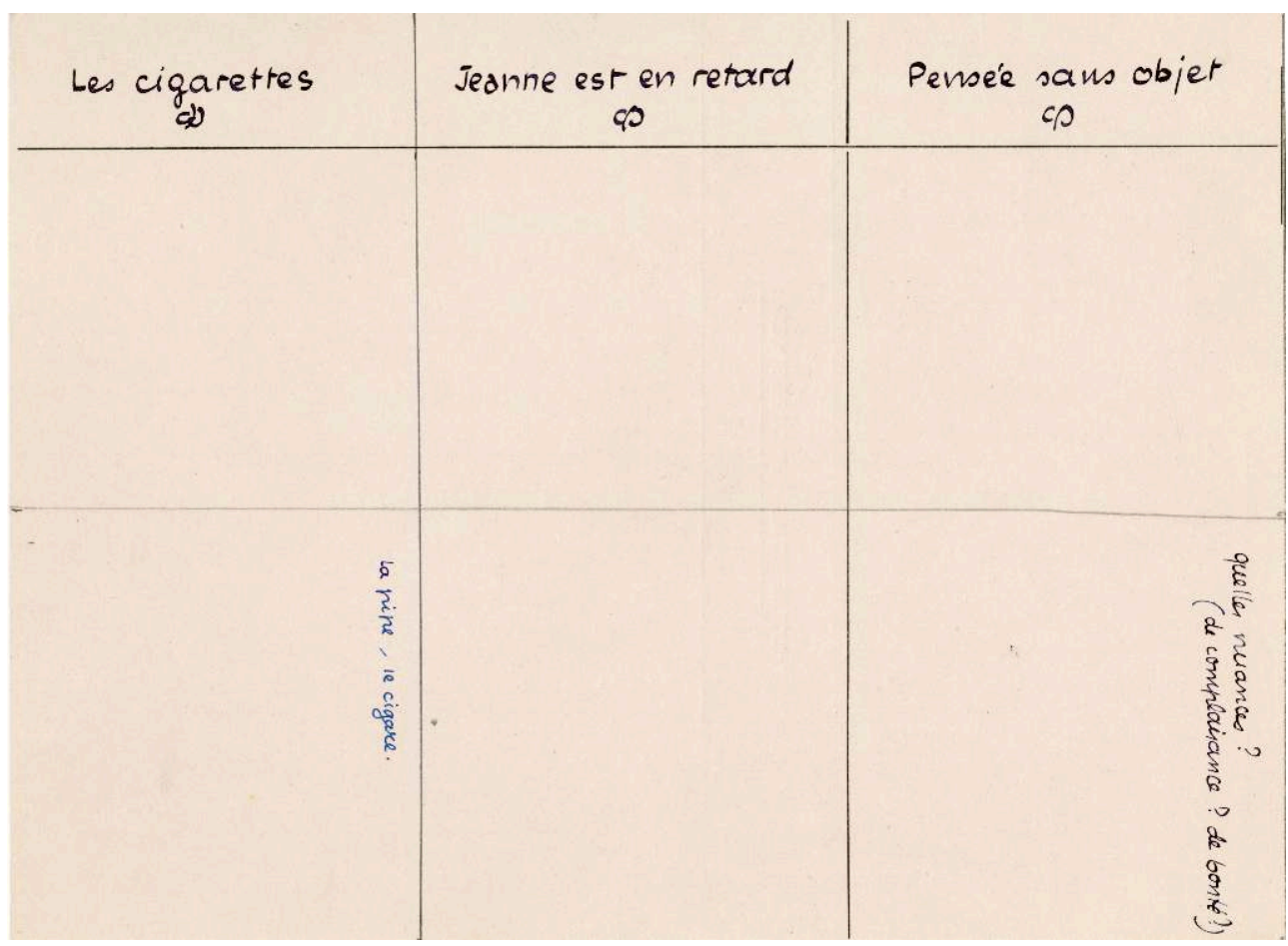

Source : fonds Paulhan, IMEC 
Figure 3. Plan des Causes célèbres : sections "Une main sous les pierres ", "Le Berger d'Ecosse », "Plaisirs perdus »

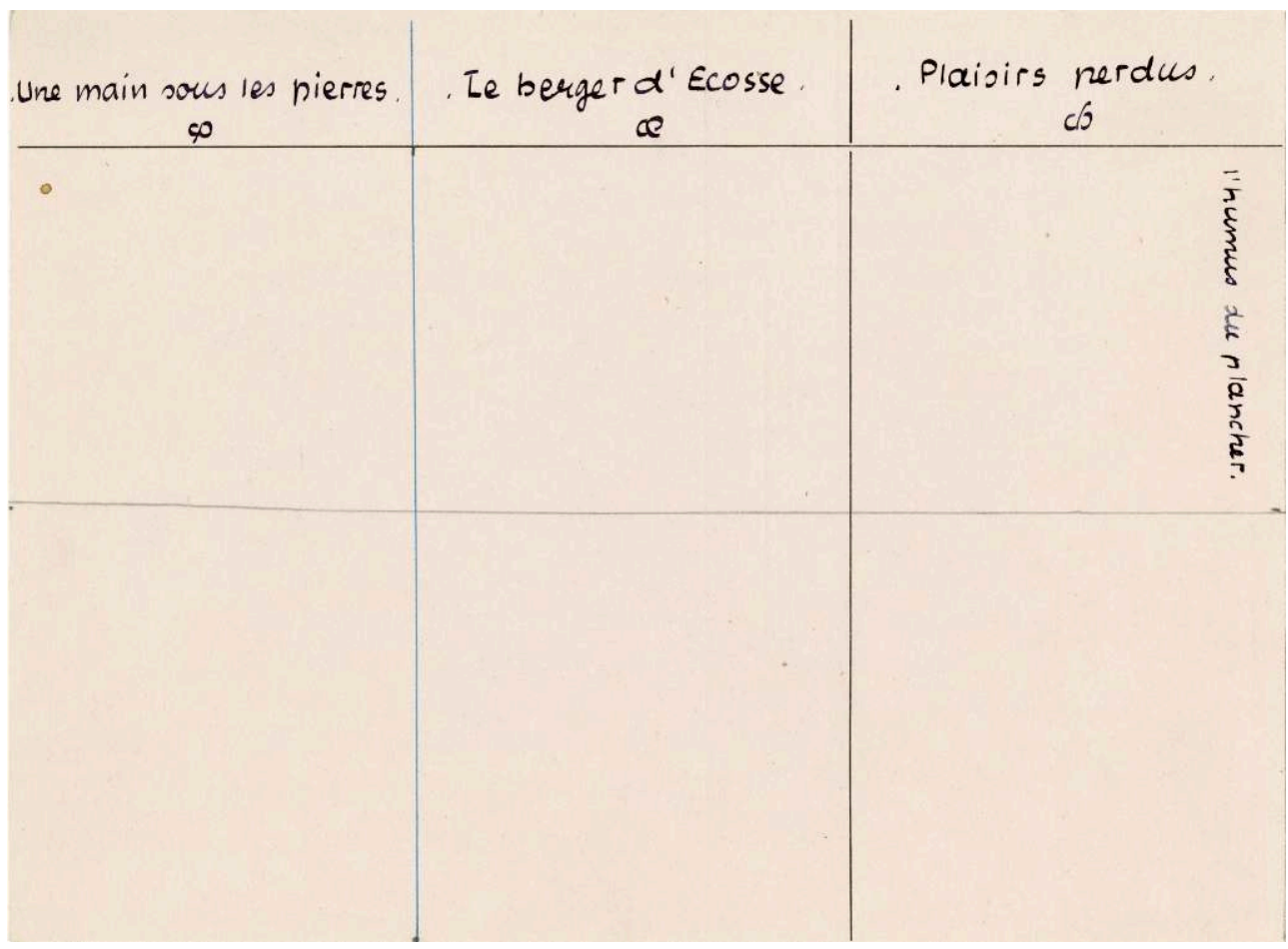

Source : fonds Paulhan, IMEC

Figure 4. Plan des Causes célèbres : sections « La petite Violette ", " La Bonne entente ", " Un rêve dans le réveil »

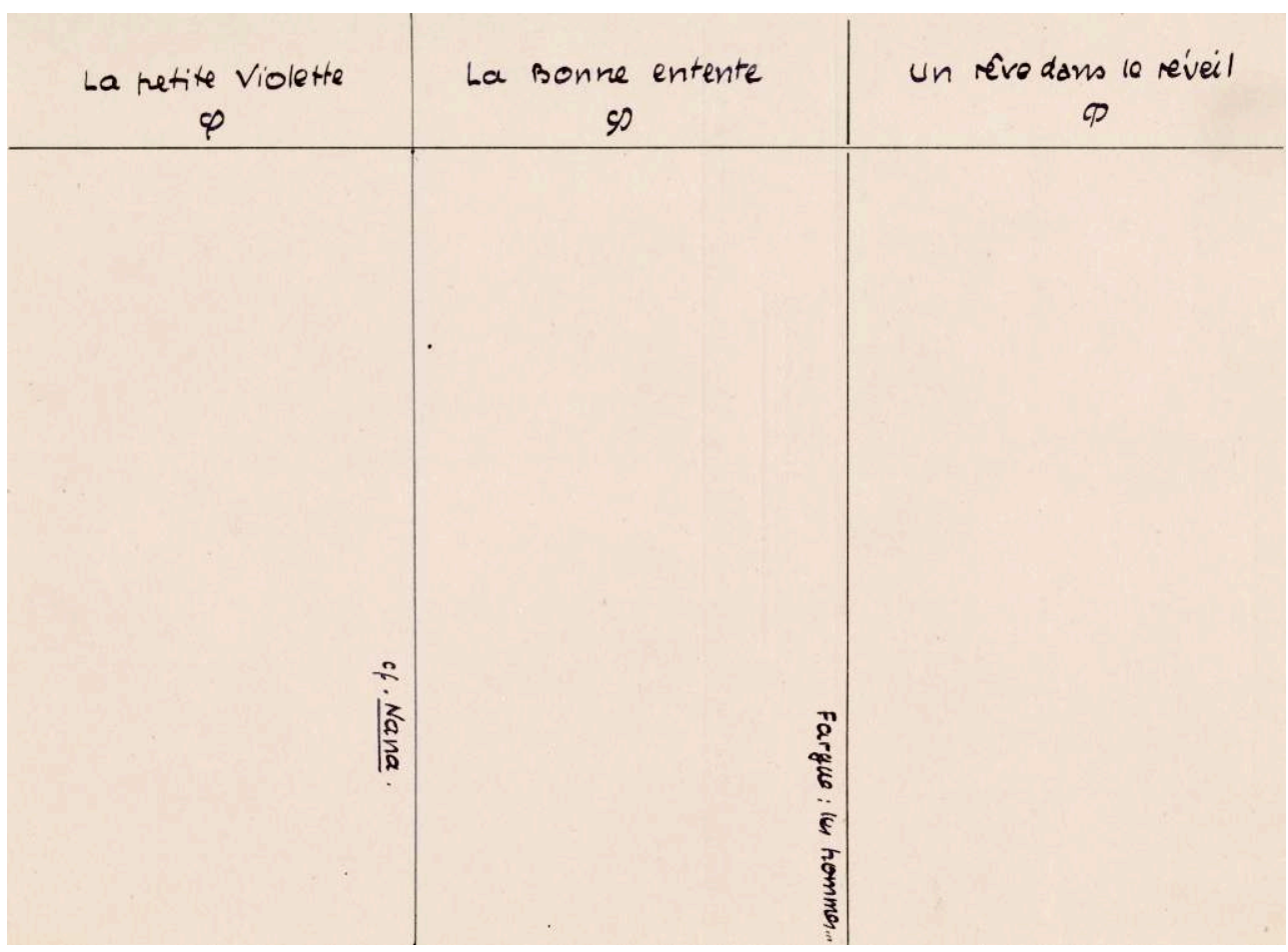

Source : fonds Paulhan, IMEC 
Le titre de section semble constituer le noyau autour duquel se déploie le texte, conçu comme un ensemble de cases vides à remplir. Comme on le voit, la plupart de ces grilles sont restées vierges. Les éléments inscrits dans les cases renvoient à des marques d'intertextualité absentes du texte définitif (Nana pour «La petite Violette ») ou des images (l'humus du plancher dans «Plaisirs perdus», qui est pourtant un développement marginal du texte, la multiplication des seins dans «Je sais ce que tu veux » qui s'intitulera « Tout feu tout flamme » dans la version définitive). Ce dispositif, s'il permet à Paulhan de nourrir petit à petit ses chapitres, ne fige pas encore l'ordre des sections. De même, il utilise de petites cartes sur lesquelles il inscrit le titre et un résumé du contenu de la section.

Figure 5. Brouillon des Causes célèbres : carte résumant le contenu de la section « Rêve dans le réveil »
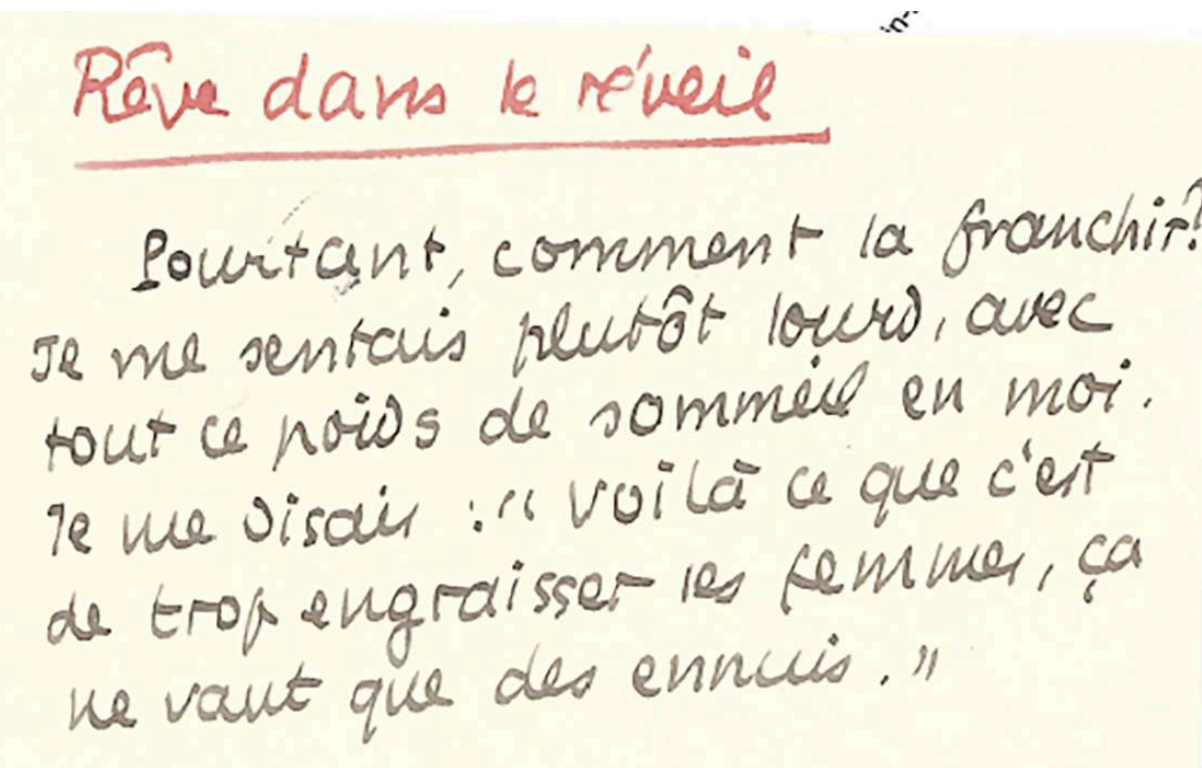

TSUP.

Source : fonds Paulhan, IMEC

Le format des petites cartes lui permet de rebattre à loisir ces différentes sections dont l'ordre n'est pas encore figé, et fait l'objet d'un questionnement, souvent mené de manière collective. Le document suivant montre ainsi la manière dont l'organisation des différentes sections au sein du texte a fait l'objet de classements concurrents, mobilisant plusieurs contributeurs, dont Fautrier, peintre et ami proche de Paulhan, dont le classement n'a finalement pas été retenu. 
Figure 6. Table des matières des Causes célèbres : organisation collective des sections

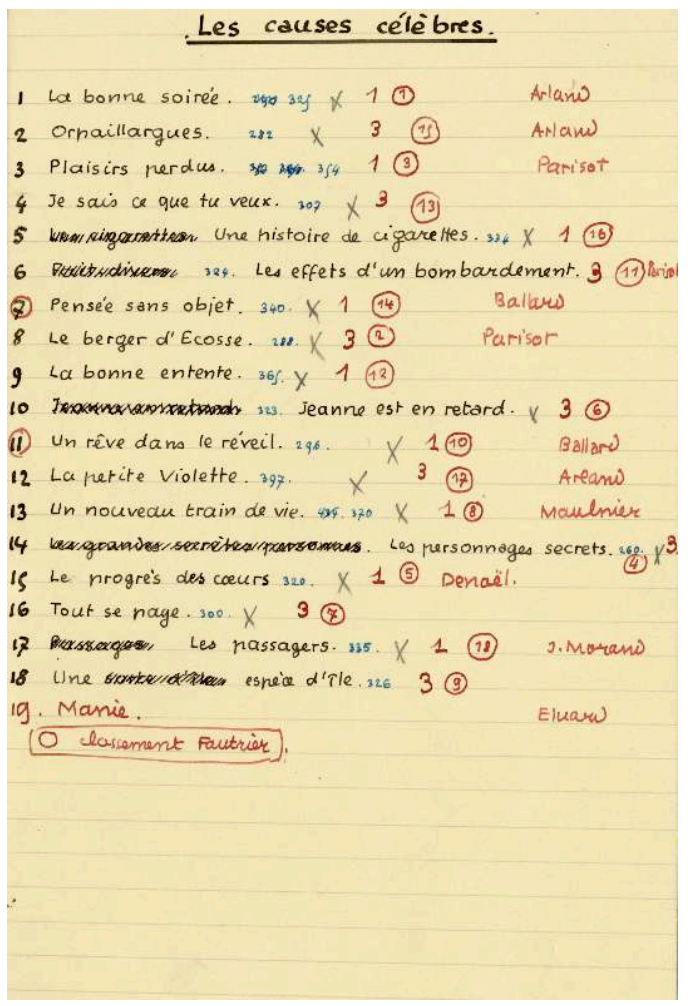

Source : fonds Paulhan, IMEC.

Il manque évidemment les archives permettant de comprendre les choix des uns et des autres, et de restituer leurs échanges et leurs arguments en faveur de tel ou tel ordonnancement. Pour Bernard Baillaud, ce procédé illustre l'inquiétude rencontrée par Paulhan au moment d'organiser la disposition de ces textes (Baillaud 2000 : 304). Il nous semble aussi qu'on pourrait voir dans cet ensemble de dispositif une entreprise qui tient à la fois de la logique (logique du récit) et du jeu collectif, puisque chaque lecteur, en réordonnant ce matériau, propose un parcours de lecture singulier.

\section{Une succession d'unités elles-mêmes divisées en sous-unités : le cas de l'hypersegmentation des textes}

$7 \quad$ Un second type de structure récurrent au sein des récits paulhaniens renvoie au cas où les textes sont divisés en sections, souvent au nombre de trois, elles-mêmes divisées en sous-sections. C'est le cas de Lalie (six sections divisées en sept et huit sous-sections numérotées) et du Guerrier appliqué (dix sections comprenant, à l'exception de la première, entre deux et trois subdivisions numérotées). Ces subdivisions sont ellesmêmes découpées par des blancs typographiques.

Le découpage le plus souvent observé est alors la structure tripartite : c'est le cas de La Guérison sévère (1919), texte dans lequel Paulhan évoque la crise de pneumonie qui l'a frappé en 1918, après qu'il a été mobilisé pendant la guerre. Les trois parties du récit sont elles-mêmes découpées en subdivisions, mobilisant différents procédés : blancs typographiques et passage à l'italique dans la première partie - fragmentation qui s'explique en partie par une écriture marquée par la fièvre ; sous-sections numérotées dans la deuxième partie, qui donnent à voir le point de vue de Juliette, l'épouse du 
narrateur, blancs typographiques à nouveau au sein de la troisième partie. Une autre ligne de fragmentation du texte se lit dans le déchirement entre deux femmes, l'épouse et la maîtresse, dont le narrateur a conservé les lettres : son point de vue reste inconnu, mais le texte met bien en place l'image du triangle amoureux.

Même Progrès en amour assez lents, dont la structure est apparemment plus linéaire, obéit en réalité à cette organisation tripartite, comme le montre le plan du récit reproduit dans l'édition de Bernard Baillaud (Paulhan [1966] 2006: 462) : si le plan se distribue en trois parties, celles-ci sont escamotées dans le texte final au profit d'une simple numérotation continue. Le plan montre en effet que le texte s'organise autour $\mathrm{du}$ récit des amours successives du narrateur avec trois jeunes filles, Jeanne, Juliette et Simone auxquelles sont consacrées respectivement trois, trois et six sections du texte. Aux amours du narrateur font d'ailleurs écho, dans la cinquième section, dont le titre a des résonances proustiennes ("L'après-midi des jeunes filles»), celles de ses deux camarades Maurice et Duffy avec Georgette et Antonine (possibles avatars de Gilberte et Albertine ?), phénomène qui souligne à nouveau l'importance des combinaisons à trois têtes au sein des récits paulhaniens. À Germaine Pascal (qui deviendra sa femme), Paulhan écrit ainsi en 1918 au cours de l'écriture du texte: "Je veux refaire complètement mes " 3 récits", leur donner une portée tout à fait nouvelle et bien plus d'unité qu'ils n'en ont. Je ne te les renverrai qu'après » (Paulhan 2006: 466). La tension entre la section et la question de l'unité du récit, essentielle chez Paulhan, atteint ici son point maximal, et est l'objet d'un profond questionnement puisque Paulhan, tout en aspirant à cette unité, tient en même temps à conserver une certaine hétérogénéité au sein du texte. Comme Bernard Baillaud l'a souligné, Paulhan entend faire imprimer en caractères différents les trois types de textes (remarques présentes, récits, lettres de Juliette et de Simone) qui forment le récit (Baillaud $2000: 353$ ). Les notes préparatoires du texte, reproduites également dans l'édition de Bernard Baillaud, insistent sur l'importance de la question de l'unité du texte aux yeux de Paulhan, et témoignent pour Bernard Baillaud, des tâtonnements avec lesquels Paulhan cherche à saisir l'unité de son œuvre. Au sein de ses notes, on trouve ainsi la mention : «Une grande unité dans le récit » (Paulhan 2006 : 474). Cette unité serait apportée grâce à ce que Paulhan appelle "l'unité autour de Jeanne », première figure féminine rencontrée dans le récit, qui aimanterait l'ensemble du texte, dans la mesure où elle l'encadre (elle réapparaît à la fin), et où, contrairement aux deux autres jeunes femmes, le narrateur la désire dès la scène du chapitre deux ${ }^{5}$. Des effets d'échos contribuent également à structurer le texte et à lui apporter son unité, en dépit du caractère apparemment fragmentaire de l'ensemble. La discontinuité de la narration qui escamote les repères temporels est renforcée par le procédé des blancs typographiques au sein des sections. On trouve ce type d'effet d'échos, et de subtil dosage entre répétition et progrès à travers la scène du premier rendez-vous du narrateur avec chacune des jeunes filles : la première ne vient pas, la seconde vient avec ses deux amies et se dérobe, la troisième le retrouve, précisant qu'elle ne porte ni corset ni ceinture, et s'offre à lui.

\section{Entre fragmentation et progression narrative : le motif de la quête dans les récits paulhaniens}

10 Comme le souligne Bernard Baillaud dans sa thèse (2000: 350$)$, la répartition en trois parties correspond moins à une simple différenciation des personnages qu'aux étapes 
d'une expérience. Le récit peut en effet se lire comme un trajet initiatique. Certes, il présente un caractère fragmenté revendiqué par l'auteur: en première page de la version dactylographiée, Paulhan présentait d'ailleurs son texte avec ces mots: "Progrès en amour assez lents, Fragments d'un récit ». (Ce sous-titre n'a pas été repris dans les éditions de 1966 et de 1968.) Pourtant, le récit suit bien un arc narratif. Le point de départ est l'insatisfaction sexuelle du narrateur : "Je n'éprouvais pas le plaisir naturel qui m'était dû » (Paulhan [1966] 2006 : 94) affirme celui-ci dans la première section du texte. Si le récit semble donc s'organiser autour d'une quête, cette dernière tire son caractère déconcertant de l'indifférence avec laquelle elle est menée. De Juliette il écrit ainsi : "ai-je pour elle la même sorte d'indifférence que je redoute d'avoir pour moi ?» (Paulhan [1966] 2006 : 112). On retrouve ces mêmes procédés de distanciation dans Le Guerrier appliqué, qui transpose l'expérience militaire de Paulhan sur le front. L'initiation patriotique comme l'initiation amoureuse sont racontées avec la même distance - d'ailleurs le narrateur des Progrès en amour prend volontiers la pose de l'élève appliqué, comme lorsque Simone l'invite à se montrer plus lent et qu'« il s'y appliquait »(Paulhan [1966] 2006: 130). Les différentes réactions des trois jeunes femmes semblent pourtant indiquer que la quête progresse. Les débuts et fins des premières sections sont le lieu où le narrateur fait le constat de ses échecs, avec des jeux d'oscillation qui soulignent l'instabilité de la narration. Ainsi au sujet de la même jeune femme, Jeanne, il écrit à la fin de la section $3:$ «j'aurais dû me rappeler tout cela avec plus de gravité. C'est que je ne prends pas assez mon parti, par une sorte d'indifférence ou de lâcheté » (Paulhan [1966] 2006: 104). On trouve à la fin de la section suivante : «Je songeais alors à Jeanne, et j'étais porté à donner trop de gravité à mon échec» (Paulhan [1966] 2006: 106). Cette quête se clôture-t-elle sur un accomplissement? À partir de l'apparition de Simone, au vocabulaire de la perte se substitue le lexique de la conquête, même si celui-ci, de nouveau, est mis à distance par des modalisateurs. Fin de la section 9: "Or si c'est là une victoire durable, je me le demande " (Paulhan [1966] 2006: 130). Début de la section 12 : «Ai-je donc été plus habile ou plus pressant que les autres fois?» (Paulhan [1966] 2006: 138). Dans la section 2, le narrateur faisait le constat d'un « défaut » qui l'entravait dans sa sexualité avec les femmes, défaut qu'il reconsidère ainsi à la toute fin du texte : "Que faire, dans la vie, d'un défaut? Il faut attendre qu'il devienne une qualité » (Paulhan [1966] 2006 : 142). Si le texte n'autorise guère de lecture univoque, il semble que la trajectoire accomplie résulte moins d'une avancée, d'un changement, que d'un renversement où le défaut s'inverse en son contraire. Par un renversement ironique caractéristique du récit paulhanien, le narrateur se voit à présent couronné de succès inattendus dus à la réputation favorable qui lui viendrait des confidences faites par Simone à ses amies : «La préoccupation où j'étais de ne pas décevoir Simone fit qu'elle était complètement heureuse alors que j'étais loin de l'être, et même demeuré plus haut assez indifférent " (Paulhan 2006: 129). Ce motif de la quête se révèle ainsi déconcertant à un double niveau: sur le plan sémantique (le caractère énigmatique de l'objet recherché et l'absence de conclusion définitive) et sur le plan de la structure, puisque Paulhan use d'une forme extrêmement fragmentée, parfois décousue, qui entre en tension avec le motif de la quête dont on attendrait, en dépit des péripéties qui l'accompagnent traditionnellement, une forme plus continue et une progression narrative plus lisible.

11 Ce jeu des permutations et des renversements est au cœur de l'organisation du texte, et de la manière dont il découpe, regroupe et isole ses différentes sections. Il engage aussi les rapports entre les sexes, dont le récit explore les différentes modalités à travers les 
amours du narrateur, plus ou moins actif, et de ses deux camarades. Une anecdote racontée par Maurice, qui la connaît par cœur (à peu près au centre du récit, section 7), illustre bien ce phénomène :

Le curé de chez nous a dit à la messe, l'autre dimanche: "Quand la messe est terminée, les jeunes gens vont courir les champs; les jeunes filles montent sur les échelles pour cueillir les cerises. Les garçons viennent par-dessous, et crient "je le vois !" Et que voient-ils, chrétiens ? La porte de l'enfer toute grande ouverte. Mais désormais il n'en sera plus ainsi... (Il fit ici une pause)... Les jeunes filles se mettront dessous et les garçons monteront dessus. » (Paulhan 2006 : 123)

Le renversement est donc à la fois un fil thématique du récit et un mode d'organisation structurel. Ce motif de la quête, qui organise la narration, est récurrent dans les récits paulhaniens, et nous allons examiner un autre cas à travers l'exemple du Pont traversé (1921).

Le Pont traversé est sûrement le texte le plus emblématique de cette structure tripartite que nous évoquions plus haut. Il se divise en effet en trois nuits, qui correspondent à trois sections du texte, elles-mêmes divisées en trois rêves, dont le narrateur fait le récit, et qui portent tous un titre. La structure du récit est encore complexifiée par un autre type de mouvement: il s'agit d'un aller-retour entre le «Je » qui enregistre les événements du récit et le «Je » qui les commente. Le cas, assez discret dans Progrès en amour, est particulièrement visible dans Le Pont traversé. Les neuf rêves dont le narrateur fait ici le récit sont donc autant d'étapes d'une quête amoureuse, tentatives pour renouer avec une figure aimée que la "gêne", les "reproches", la "rancune " ont éloignée. Divisé en trois sections qui correspondent à trois nuits, le récit est en réalité structuré par deux mouvements: l'un est un mouvement de progression, d'avancée, comme le titre le laisse attendre, le texte se lisant alors comme un trajet initiatique dont le narrateur enregistre les progrès, l'autre, un mouvement de va-etvient entre le rêve et son commentaire éveillé, lisible dans la structure typographique du texte puisque l'auteur utilise le romain pour les récits de rêve, l'italique pour la phase d'éveil où il s'en ressaisit pour les commenter. Chaque section se compose donc à la fois des récits de rêve, et en début et clôture de chaque section (voire, pour certaines, entre les récits de rêve) de paragraphes en italique qui relèvent du commentaire du narrateur.

Jean-Yves Tadié observe ainsi que « la narration des récits paulhaniens se déroule sous le signe du dédoublement entre un moi-spectacle et un je-narrateur qui a la charge de l'observation et plutôt que de l'explication, de l'interrogation" (Tadié 1994: 20). Ce dédoublement est sensible dès la première phrase du texte : "À peine eus-je pris la décision de te rechercher, que je me répondis par une abondance de rêves » (Paulhan [1921] 2006 : p. 85). La phrase lance bien le leitmotiv de la quête. Comme on le voit, la construction de la phrase dresse un parallèle entre le «Je » rationnel, du côté de la décision, et le " Je » réceptacle des rêves : le dialogue entre ces deux instances du «Je ", que les changements dans la typographie matérialiseront, se lit dans la forme réfléchie "Je me ». La formule utilisée par Jean-Yves Tadié semble d'autant plus bienvenue au sujet du Pont traversé, que la fin de chaque section est dramatisée par la mention « Fin de la première nuit ", " Fin de la seconde nuit ", « Fin de la troisième nuit ": chaque nuit résonne ainsi comme un acte composé de trois scènes - les rêves -, au sein de la pièce de théâtre dont le «Je » est le spectateur. Le tomber de rideau entre chaque acte ne correspond paradoxalement ici pas à la plongée dans l'obscurité mais à l'éveil et à l'arrivée du jour. 


\section{La structure tripartite reine ? Construction et déconstruction du plan}

14 On retrouve le même type de construction en trois unités elles-mêmes divisées en trois sous-sections dans Les Douleurs imaginaires (1953). Si ce texte ne figure pas dans le volume consacré aux récits paulhaniens de l'édition de Bernard Baillaud, il comprend néanmoins des éléments narratifs. À partir de son expérience de la maladie (une crise de sciatique) et de son récit des différentes tentatives pour se guérir (la visite chez l'acupuncteur, censée l'avoir guéri), le narrateur développe une méditation philosophique sur le réel et l'illusion, qui s'inscrit dans le cadre plus général de la réflexion que Paulhan mène sur le langage. Chacune de ces trois parties se clôt sur une section intitulée "marges ", en italique, où Paulhan se ressaisit du matériau rassemblé lors de ces observations dans une perspective plus explicitement philosophique.

Comme c'était le cas dans Le Pont traversé, le texte se structure en deux mouvements : un arc narratif autour de la crise de sciatique et des tentatives de guérison. Le texte s'achève sur le constat que les diverses maladies qui affligent le narrateur ont cédé avec l'âge. Un autre mouvement réside dans le va-et-vient entre l'expérience de la maladie et la réflexion à laquelle elle donne lieu, qui se poursuit dans ce qui est identifié comme «les marges» du texte en prenant le point de départ suivant: la maladie, et les changements de perception qu'elle induit, est-elle un instrument permettant de prouver la réalité du monde extérieur? Nombreuses sont les références explicites et implicites concentrées dans ce très court texte, depuis Descartes à Proust (la maladie redéfinit les contours du monde puisqu'elle modifie la perception). Les archives donnent à voir des plans méticuleusement tracés. 
Figure 7. Plan des Douleurs imaginaires sur une carte

\section{LES DOULEURS IMAGINAIRES}

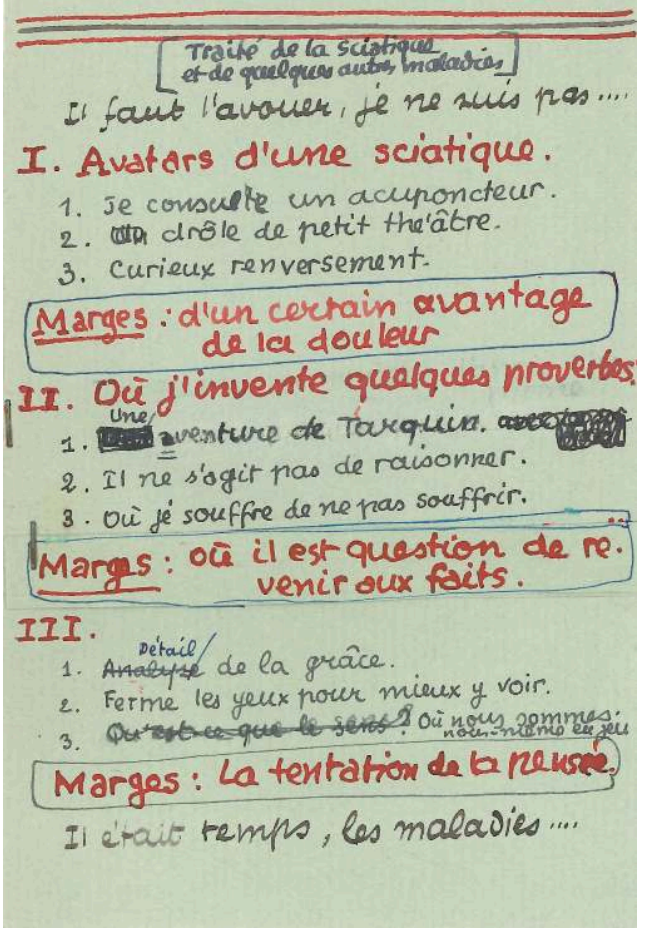

Source : fonds Paulhan, IMEC.

Figure 8. Plan détaillé des Douleurs imaginaires, avec adjonction sur papier collé

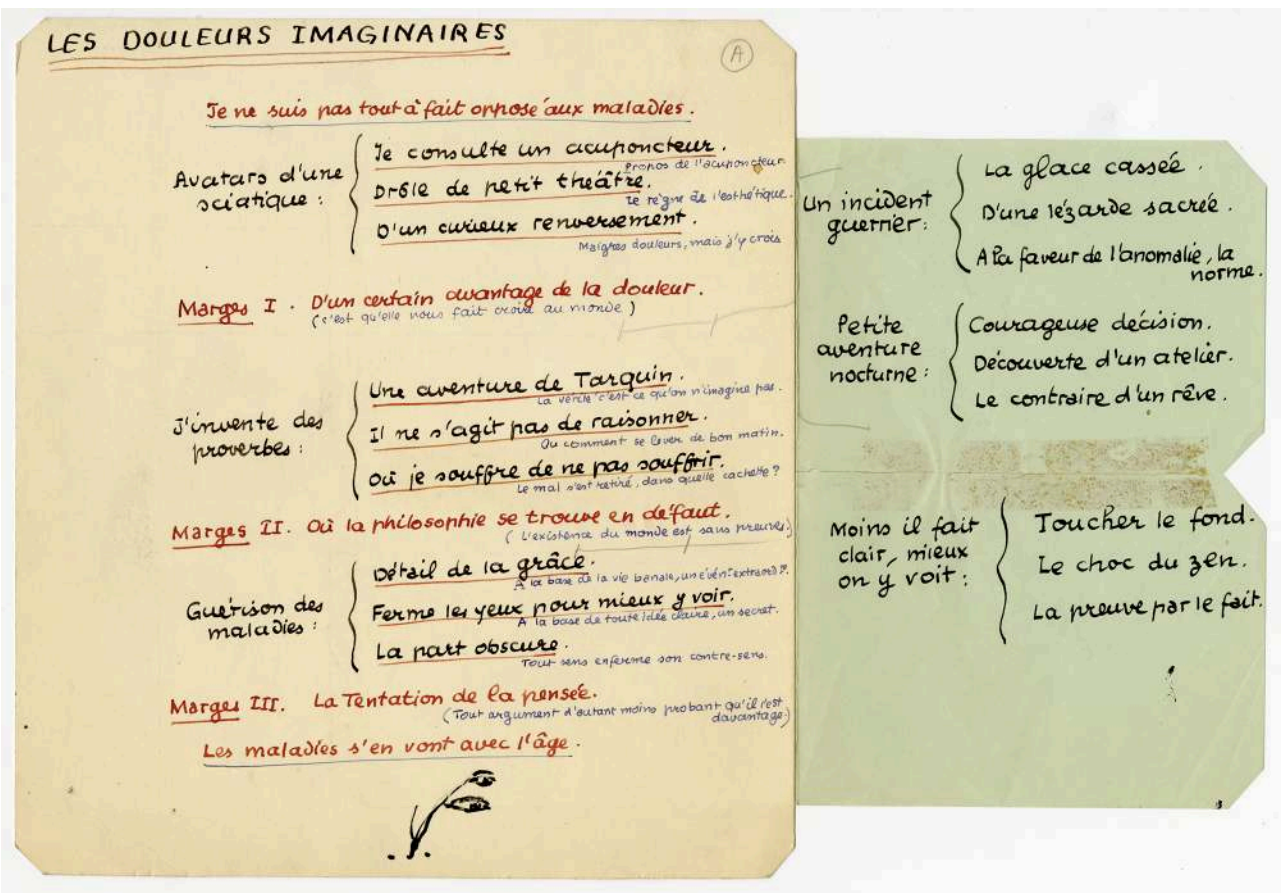

Source : fonds Paulhan, IMEC

Ces documents pourraient être l'œuvre d'un khâgneux particulièrement méthodique s'ils ne traduisaient pas le goût de Paulhan pour l'organisation très visuelle de son 
propos, et pour l'ornementation de ses plans. On repère différents procédés : couleurs, graphies, numérotation, encadrés, etc.

D'autre part, malgré la fascination qu'exerce visiblement sur Paulhan la machine bien huilée de la structure tripartite, les différents plans qu'il échafaude ne se contentent pas de décliner la même structure et d'en renommer les sections. L'écriture se constitue en bâtissant différentes formes de plan, qui réordonnent le matériau du texte et le rebattent en l'appréhendant selon différentes perspectives, comme si cet effort de construction et déconstruction du plan permettait de donner naissance à l'écriture. Un autre plan des Douleurs imaginaires traite ainsi à part les faits et les marges (qui sont, nous l'avons vu, réparties au sein de chaque section), réarticulant l'ensemble autour de ces deux grandes parties, qui semblent alors pouvoir être traitées de manière autonome.

Figure 9. Plan des Douleurs imaginaires, réorganisé entre « les faits » et « les marges »

\section{LES DOULEURS IMAGIIMAIRES}

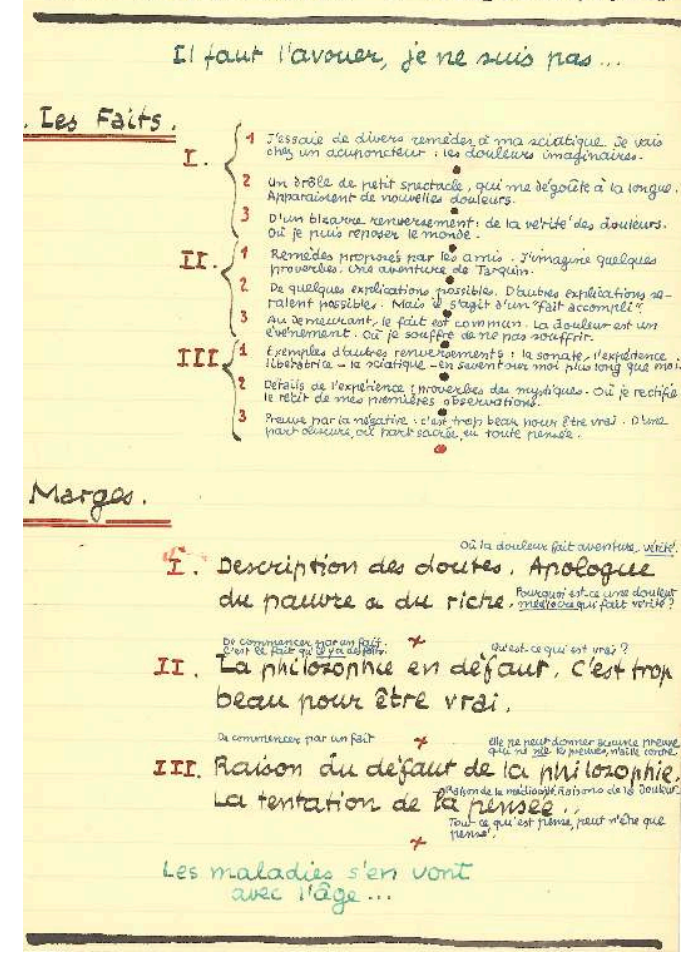

Source : fonds Paulhan, IMEC.

Un autre document de travail montre la manière dont s'ébauche le raisonnement. 
Figure 10. Brouillon des Douleurs imaginaires : première partie (d'une part)

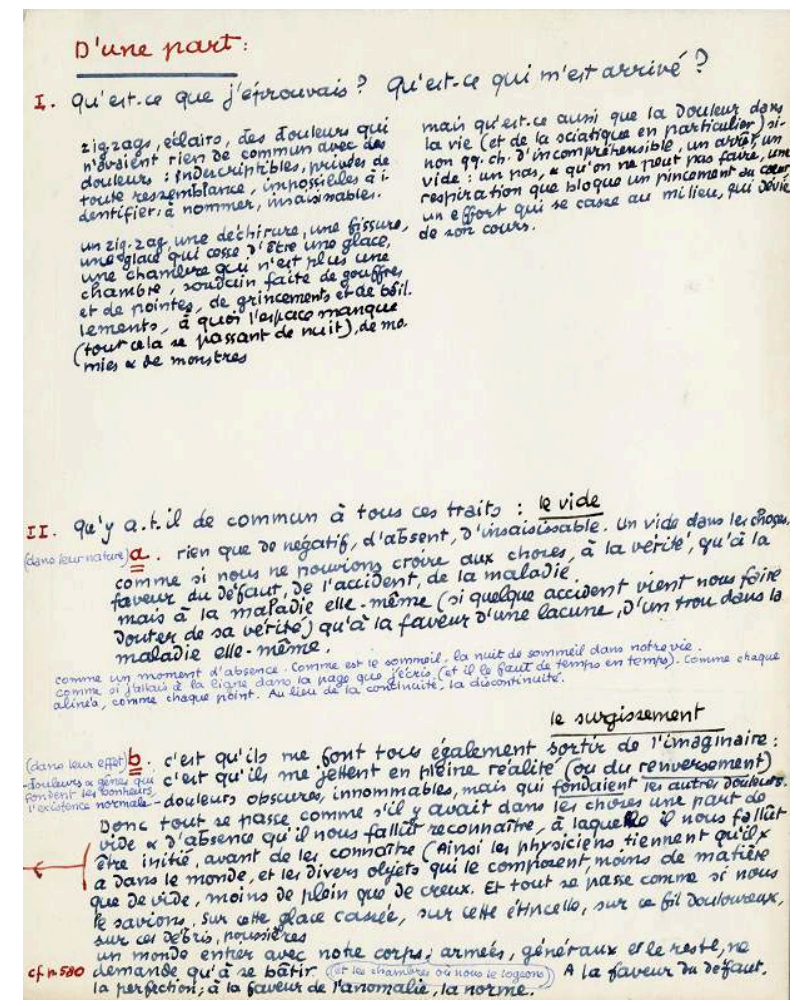

Source : fonds Paulhan, IMEC.

Figure 11. Brouillon des Douleurs imaginaires : deuxième partie (d'autre part)

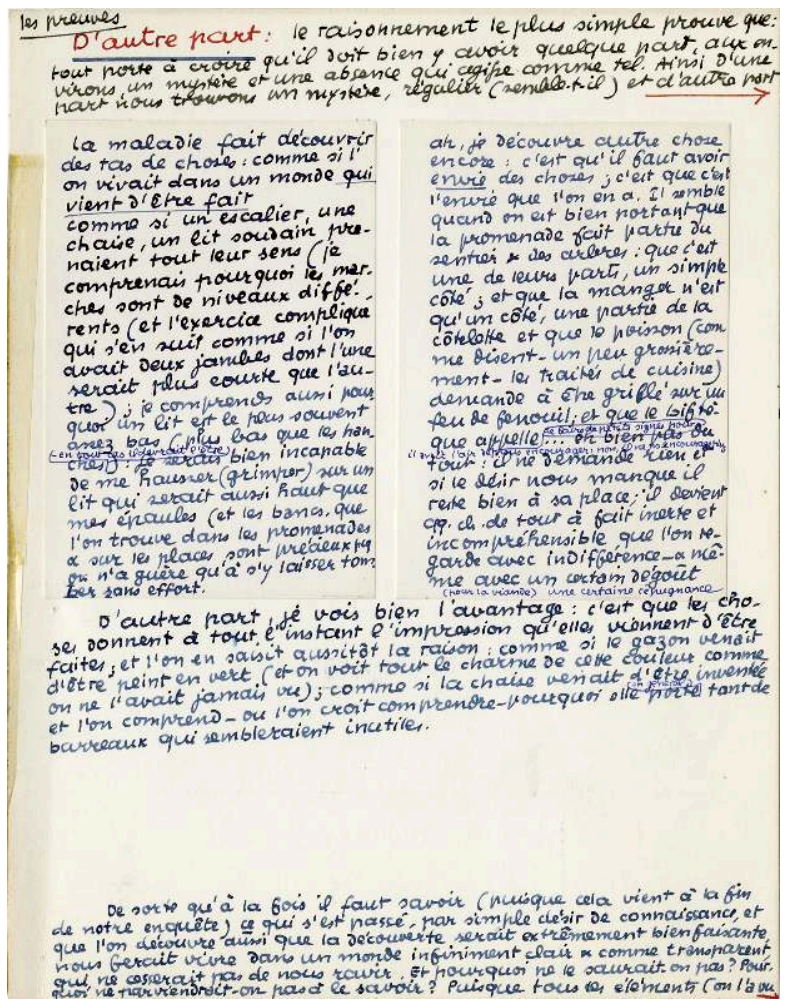

Source : fonds Paulhan, IMEC 
Le raisonnement se découpe en deux parties bien distinctes (d'une part, d'autre part) ; elles-mêmes subdivisées en sous-sections soigneusement numérotées. Le premier point (I) se compose des éléments qui renvoient à deux expériences corporelles éprouvées par Paulhan: la douleur de la sciatique et la traversée de sa chambre méconnaissable dans l'obscurité ; le deuxième point (II) détaille les points communs que Paulhan dégage entre ces deux expériences, soit le vide (c'est le défaut, la lacune qui nous permet de croire aux choses) et le surgissement (ces événements qui nous font sortir de l'imaginaire et nous jettent en pleine réalité). En réalité, Paulhan évoque ici deux épisodes. L'un - l'expérience de la sciatique - figure bien, nous l'avons vu, dans Les Douleurs imaginaires. L'autre est relaté dans Le Clair et l'Obscur (1958) et renvoie également à la question de la modification de la perception : rentrant dans sa chambre obscure, la nuit, le narrateur, qui craint de réveiller sa femme, donne un rapide coup de lumière lui permettant de repérer les obstacles et de gagner son lit. Le stratagème ne suffit pas à lui permettre de trouver son chemin : à la familiarité des lieux se substitue un paysage mental mouvant, étrange, instable. Comme dans le cas de la douleur, la réflexion se noue autour de la question suivante: quelle expérience fait-on du réel quand notre perception est modifiée, et que nous nous trouvons, à cause de la maladie ou de l'obscurité, privés de nos repères habituels? Du point de vue de la construction du texte, les archives montrent que les deux essais étaient d'abord conçus pour ne former qu'un seul texte.

Figure 12. Plan des Douleurs imaginaires comprenant des sections du Clair et l'Obscur

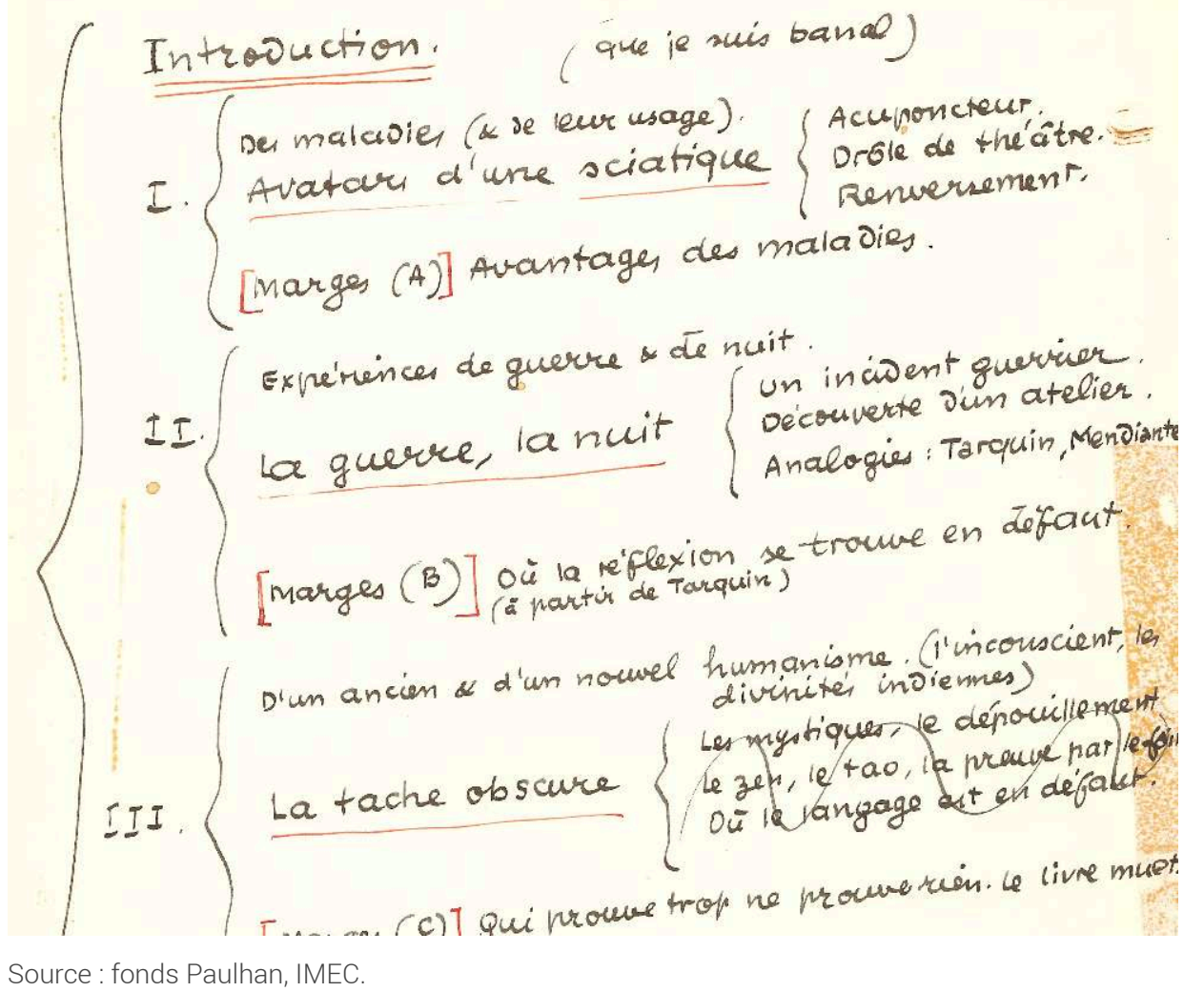

Sur l'image ci-dessus, le découpage opéré par Paulhan montre que si la section I correspond à la première partie des Douleurs imaginaires, la section II renvoie en réalité au deux premiers chapitre du Clair et l'obscur, texte constitué de six chapitres divisés en 
trois sections. Si l'on se reporte à la figure 8 , on s'aperçoit qu'à chacune des sections des Douleurs imaginaires, correspondent les trois premiers chapitres du Clair et l'obscur. Comme si ce dispositif de découpage des textes et de rebattement incessant de leurs différentes sections, remaniées non seulement au sein des textes eux-mêmes, mais aussi, comme on le voit, d'un texte à l'autre, guidait et nourrissait le processus d'écriture, organisant non seulement l'unité d'un texte, mais de l'œuvre tout entière.

\section{Conclusion}

Cette extrême segmentation narrative présente donc, à notre sens, une double signification. Elle est tout d'abord une mise à l'épreuve du lecteur. Bernard Baillaud (2000 : 293) souligne ainsi l'absence de table des matières dans l'édition Sansot (1917), du Guerrier appliqué, texte que nous évoquions plus haut. Le lecteur est donc amené à cheminer au sein du récit sans avoir sous les yeux la disposition complète du livre. Pour reprendre les termes de Bernard Baillaud, «il ne s'agit pas d'un secret, soluble en une formule unique et qui serait cachée dans la disposition du livre, mais d'un dispositif textuel orienté vers un processus de lecture» (Baillaud 2000 : 297). Processus qui demande le concours du lecteur, et dont le parcours n'est jamais unique. D'autre part, cette segmentation a aussi un sens au sein de l'œuvre entière, puisque les différents fragments qui composent les récits paulhaniens, mais aussi les textes de nature essayistique, entrent en résonance les uns avec les autres. Ainsi, la structure d'un récit comme le Pont traversé est sensiblement la même que celle des Douleurs imaginaires, de nature plus essayistique. D'un point de vue thématique les deux textes se rejoignent également à travers la mise en scène d'un " Je » spectacle (c'est d'ailleurs le titre d'une des sections des Douleurs imaginaires : "Drôle de petit théâtre »), réceptacle de diverses sensations et émotions (douleur et rêves), même si dans Les Douleurs imaginaires, l'observation donne lieu à une réflexion de nature philosophique.

Comme on le voit, il existe une unité forte et des effets de circulation entre des textes de nature fort différente, du récit à l'essai. Nous reprenons à nouveau une formule de Bernard Baillaud au sujet du Guerrier appliqué, selon qui les différents segments du texte présentent une "disponibilité " pour d'autres orientations que celles que le récit contient (Baillaud $2000: 301$ ). Ce phénomène renvoie aussi à la contradiction entre la fascination pour les structures rigoureuses héritées de la rhétorique classique d'un côté, et l'impossibilité de conclure de l'autre, avec la volonté de laisser le sens battant et l'œuvre en devenir. Si l'on réutilise la formule de Bernard Baillaud à l'échelle de l'ensemble de l'œuvre, ces différents segments sont potentiellement disponibles pour d'autres usages, dans d'autres textes. On touche là, nous semble-t-il, un point essentiel au sein de l'œuvre paulhanienne, à savoir la tension entre le fragment et l'unité de l'œuvre, question centrale pour Paulhan, qui prend un tour encore plus vif après la Seconde Guerre mondiale où la question de l'unité se charge de résonances à la fois littéraires, morales et politiques, avec la volonté de rassembler une communauté intellectuelle déchirée par les dissensions héritées de la guerre. Rappelons aussi que ses œuvres complètes seront publiées à partir de 1966 chez Tchou. Dans une lettre non datée (1953 ou 1954) il écrit ainsi à Arland aux côtés de qui il a relancé La NRF en 1953 : "Je travaille; Ah, il m'arrive d'être jaloux de la perfection, chacune en soi, de tes œuvres; Moi je suis loin d'avoir achevé celle qui justifierait les autres. Mais il me semble qu'avec les Douleurs imaginaires, je fais un pas sérieux dans ce sens ${ }^{6}$. » 
21 À l'œuvre arlandienne, présentée comme un ensemble de textes dotés d'une existence autonome, il oppose sa propre œuvre conçue comme un ensemble organique, en construction, qui prendrait sens au sein d'un ordonnancement général. Ce propos rejoint la quête d'unité menée par Paulhan tout au long de son œuvre, et à travers son œuvre: pour Bernard Baillaud, cette quête voit se substituer le vocabulaire de la métaphysique à celui de la segmentation. Au sujet du Guerrier appliqué, Paulhan écrit ainsi à Albert Uriet en 1916: "Je voudrais bien savoir si ça fait corps ensemble. Je voudrais accentuer, dans chaque chapitre, une intrigue matérielle, de hasard - et dans l'ensemble une sorte d'âme (de métaphysique) de la guerre " (cité dans Baillaud 2000 : 303). À l'intrigue matérielle, du côté du fragment, du chapitre, répond, « l'âme » du côté de l'ensemble, de la totalité. Cette idée semble chère à Paulhan, qui y revient, dans une lettre à Uriet, camarade des années de guerre, lettre citée par Bernard Baillaud et où Paulhan évoque sa thèse sur la dialectique des proverbes: «Ma thèse sera belle. Imparfaite encore, en tronçons. Mais j'y serre, vers la fin, quelque chose qui est peutêtre l'âme ${ }^{7}$.» Cette dialectique entre les fragments, les tronçons, et l'unité que constitue non seulement le texte qui l'embrasse, mais l'œuvre tout entière qui les rebat, est au cœur de l'écriture paulhanienne et de la quête qui l'anime. La multiplicité des plans, des manières d'ordonner, et de redisposer les textes afin que les différentes sections et sous-sections qui en composent la trame prennent corps et fassent âme en témoignent.

\section{BIBLIOGRAPHIE}

Baillaud, Bernard, 2000, La pensée et la fiction dans les récits de Jean Paulhan (1904-1921), thèse de doctorat soutenue à Paris-Sorbonne.

Dieudonné, Julien, 2001, Les récits de Jean Paulhan, Paris, Champion.

Murat, Michel, 2014, « Progrès dans le roman assez lents », dans Clarisse Barthélémy (dir.), La littérature selon Jean Paulhan, Paris, Classiques Garnier, p. 135-151.

Tadié, Jean-Yves, 1994, Le récit poétique, Paris, Gallimard.

\section{CEuvres de Jean Paulhan étudiées dans l'article}

Progrès en amour assez lents, 1916, dans Jean Paulhan, 2006, CEuvres Complètes, I, Récits, Paris, Gallimard, p. 89-142

La Guérison sévère, 1917, dans Jean Paulhan, 2006, Euvres Complètes, I, Récits, Paris, Gallimard, p. 217-235.

Le Pont traversé, 1921, dans Jean Paulhan, 2006, Euvres Complètes, I, Récits, Paris, Gallimard, p. $143-161$.

Les Causes célèbres, 1946, dans Jean Paulhan, 2006, Euvres Complètes, I, Récits, Paris, Gallimard, p. 275-320. 
Guide d'un petit voyage en Suisse, 1947, dans Jean Paulhan, 2006, Euvres Complètes, I, Récits, Paris, Gallimard, p. 321-349.

Les Douleurs imaginaires, 1953, dans Jean Paulhan, CEuvres complètes, t. III, Paris, Cercle du livre précieux, p. 310.

Le Clair et l'Obscur, 1958, Paris, impr. de Crété.

\section{NOTES}

1. Voir Baillaud (2000), Dieudonné (2001), Murat (2014) et Tadié (1994).

2. «Contes cruels, contes symboliques, contes logiques, histoires obscures, Les Causes célèbres ne permettent à aucun moment au lecteur de se satisfaire de l'intrigue, parce que celle-ci est machinée de manière à poser toujours le problème du sens » (Tadié 1994 : 137).

3. "C'est la structure même qui organise les récits de Paulhan (proche en cela de Bataille, qui utilise constamment l'oxymoron), et d'où il tire sa force poétique » (Tadié 1994 : 136).

4. L'Institut Mémoires de l'édition contemporaine, situé à Saint-Germain-la-BlancheHerbe, près de Caen, détient parmi de très nombreux fonds consacrés aux acteurs de l'histoire littéraire du $\mathrm{xx}^{\mathrm{e}}$ siècle, le fonds Jean Paulhan, dont les nombreuses archives éclairent à la fois l'œuvre de Jean Paulhan, et la vie littéraire du siècle, à laquelle Paulhan a abondamment participé.

5. Ces notes préparatoires montrent en effet que Paulhan, concernant ce récit en tout cas, utilise bien le terme de chapitre pour nommer les sections de son texte (Paulhan $2006: 474)$.

6. Lettre inédite de Jean Paulhan à Marcel Arland, non datée, Bibliothèque littéraire Jacques Doucet.

7. Lettre du 20 avril 1919 (date forgée), citée par Bernard Baillaud (2000: 304).

\section{RÉSUMÉS}

L'examen des notes préparatoires, des plans et brouillons conservés dans le fonds Paulhan à l'IMEC traduit l'importance accordée par ce dernier à la structuration minutieuse de ses textes. Découpés selon différents types de dispositifs, ceux-ci présentent tous une structure hypersegmentée, divisée en unités de très petites tailles. La construction du texte s'appuie sur une logique méthodique, qui voit sections et autres subdivisions s'enchaîner selon un ordre rigoureux, objet de remaniements nombreux. Cette logique de construction à la fois ludique et rigoureuse constitue une clé possible de compréhension des récits paulhaniens, dont maints observateurs ont souligné le caractère hermétique. En nous appuyant sur ces archives, nous entendons proposer une réflexion sur la structure des textes paulhaniens, sur le rôle essentiel de 
celle-ci pour la conduite et le sens même du récit, mais aussi sur la tension entre ces textes au caractère extrêmement fragmenté et le désir très fort d'unité qui habite l'ensemble de l'œuvre.

The multiple notes and outlines that can be found in the Paulhan archive in the Institut Mémoire de l'édition contemporaine (IMEC) are a testimony of his accute sense of structure, and shows how important it was in the conception of his works. Although his plans take various forms, they are all divided down to the most minute sections. The logical, sometimes dialectic construction is both rigorous and flexible, as it can be reshaped numerous times. There is even an element of play in the way plans evolve. The structure can help understand texts that generally recognised as particularly hermetic. In this article, we will study the way structure contributes to meaning, but also examine the tension that arises between a strong desire for unity and a plan that has a tendency to divide the thought process indefinitely.

\section{INDEX}

Keywords : Paulhan (Jean), table of contents, archives, plan, structure, narrative structure Mots-clés : Paulhan (Jean), table des matières, archives, plan, structure, fragments

\section{AUTEUR}

\section{CAMILLE KOSKAS}

Sorbonne Université (Labex OBVIL) 